\title{
WAVELETS FOR NONLINEAR SYSTEMS
}

\author{
F. Chaplais ${ }^{1}$
}

\begin{abstract}
We investigate how the stucture of multiresolution approximations, which are intimately related to wavelets, can be preserved through the use of a product operator. It appears that the dilatation or subsampling operator is best replaced by a smoothing operator at the nodes. Examples of related "wavelets" are given.
\end{abstract}

Keywords: wavelets, multiresolution approximation, nonlinear systems, Hermite interpolation, Hermite spaces.

\section{Introduction}

Wavelets have emerged during the last decade as a new, efficient tool for signal proceesing. They are particularly suited to the study of transients and of the local regularity of signals, with many practical applications.

In the control community, the shortcomings of the Fourier analysis, as far as time varying or nonlinear systems are concerned, are well known. One reason (not the only one) lies in its inability to represent local behaviours. It is then natural to study the use of wavelets as an alternative.

Wavelets are very closely linked to multiresolution approwimations (see section 2). In this paper, the concept of multiresolution approximation spaces is extended to multiresolution approximation albegras which provide an signal analysis that is compatible with polynomial operators. This is the necessary first step towards the design of signal processing tools that fit the needs of nonlinear analysis.

It is first proved that the classical notion of multiresolution approximation cannot provide multiresolution algebras, except for the case of piecewise constant functions. An alternative notion is proposed, which builds a sequence of nested approximation algebras. It is operational and has the same approximation properties (and more) as its classical counterpart. The difference is that the resolutions are not defined by a time rescaling, but by an explicit smoothing of the signal in a piecewise polynomial representation. The paper ends

\footnotetext{
${ }^{1}$ Centre Automatique et Systèmes, École Nationale Supérieure des Mines de Paris, 35 rue Saint Honoré, 77305 Fontainebleau Cedex FRANCE, e-mail: chaplais@cas.ensmp.fr
}

with some indications on the nonlinear equivalents of wavelets, as they can be built from multiresolution algebras.

\section{Wavelet background}

The reader may refer to [2], [4] for a clear and extensive introduction to wavelets. We shall introduce them by the way of multiresolution approximations ([5], [6]).

Definition 1 (Multiresolutions) $A$

sequence $\left\{\mathbf{V}_{j}\right\}_{j \in \mathbb{Z}}$ of closed subspaces of $\mathbf{L}^{\mathbf{2}}(\mathbb{R})$ is a multiresolution approximation if and only if, for any $j \in \mathbb{Z}$, $\left(\mathbf{V}_{j+1} \subset \mathbf{V}_{j}\right)$ and $\left(f \in \mathbf{V}_{j} \Leftrightarrow f(t / 2) \in \mathbf{V}_{j+1}\right)$, for any $k \in \mathbb{Z}\left(f(t) \in \mathbf{V}_{j} \Leftrightarrow f\left(t-2^{j} k\right) \in \mathbf{V}_{j}\right)$, the intersection of the spaces is zero in $\mathbf{L}^{\mathbf{2}}(\mathbb{R})$, their union is dense in $\mathbf{L}^{\mathbf{2}}(\mathbb{R})$, and their exists $\phi$ such that $\{\phi(t-n)\}_{n \in \mathbb{Z}}$ is a Riesz basis of $\mathbf{V}_{0}$. A biorthogonal multiresolution appxoximation is a pair of multiresolutions $\left\{\mathbf{V}_{j}\right\}_{j \in \mathbb{Z}}$ and $\left\{\mathbf{V}_{j}^{*}\right\}_{j \in \mathbb{Z}}$ such that $<\phi(t-n), \phi(t-k)>=\delta_{n, k}$.

$\phi$ and $\phi^{*}$ are scaling functions. For a given pair $\left(\left\{\mathbf{V}_{j}\right\}_{j \in \mathbb{Z}},\left\{\mathbf{V}_{j}^{*}\right\}_{j \in \mathbb{Z}}\right)$, there exists a pair of wavelets $\left(\psi, \psi^{*}\right)$, such that $\left\{\psi\left(2^{-j} t-n\right)\right\}_{n \in \mathbb{Z}}$ and $\left\{\psi^{*}\left(2^{-j} t-\right.\right.$ $n)\}_{n \in \mathbb{Z}}$ are Riesz basis of the detail spaces $\mathbf{W}_{j}$ and $\mathbf{W}_{j}{ }^{*}$ defined by $\mathbf{V}_{j} \oplus \mathbf{W}_{j}=\mathbf{V}_{j-1}$ and $\mathbf{V}_{j}^{*} \oplus \mathbf{W}_{j}^{*}=\mathbf{V}_{j-1}^{*}$, with $\mathbf{W}_{j}$ orthogonal to $\mathbf{V}_{j}^{*}$, and $\mathbf{W}_{j}^{*}$ to $\mathbf{V}_{j}$. $\phi$ and $\phi^{*}$ can be designed to have a compact support, which we shall assume hereafter. $\psi$ and $\psi^{*}$ can be then chosen to have compact support. Note that, since $\phi$ has to be of non zero average, $\psi^{*}$ necessarily has one vanishing moment (or more); hence the term "wavelet".

Denote, for a function $x, x_{j, n}(t)=2^{-j / 2} x\left(2^{-j} t-n\right)$. Then any signal $f \in \mathbf{L}^{\mathbf{2}}(\mathbb{R})$ can be decomposed into $f=\sum_{J \in \mathbb{Z}}<f, \phi_{J, n}^{*}>\phi_{J, n}+\sum_{j \leq J} \sum_{k \in \mathbb{Z}}<f, \psi_{j, n}^{*}>$ $\psi_{j, n}$. The first summation is the projection $P_{J} f$ of $f$ into $\mathbf{V}_{J}$, orthogonally to $\oplus_{j \leq J} \mathbf{W}_{j}{ }^{*}$.

The following theorem [9] jusfies the "approximation" denomination. We assume here the existence of a biorthogonal multiresolution with compactly supported scaling functions and wavelets. 
Theorem 1 (Fix-Strang) Let $p \in \mathbb{N}$. The following three conditions are equivalent

- for any $0 \leq k<p$, there exists a polynomial $\theta_{k}$ of degree $k$ s.t. $\sum_{n=-\infty}^{+\infty} n^{k} \phi(t-n)=\theta_{k}(t)$

- $\psi^{*}$ has $p$ vanishing moments

- $\exists C\left(\forall f \in H^{p+1}(\mathbb{R})\right)(\forall j \leq 0) \quad\left\|P_{j} f-f\right\|_{L^{2}} \leq$ $C 2^{j(p+1)}\left\|f^{(p+1)}\right\|_{L^{2}}$

where $H^{p+1}$ is the Sobolev space of functions with $p+1$ derivatives in $\mathbf{L}^{\mathbf{2}}(\mathbb{R})$.

We see that, when the family $\mathbf{V}_{j}$ is a sequence of approximation spaces of order $p+1$. The relation $\left(\mathbf{V}_{j+1} \subset \mathbf{V}_{j}\right)$ is qualified as a causality property. It means that, if we do some linear computations over some elements of $\mathbf{V}_{j+1}$, they will remain valid when switching to the finer resolution context $\mathbf{V}_{j}$.

The aim of this paper is to extend this causality property to nonlinear operations, e.g., the product of signals.

Let us illustrate the importance of this causality principle for the product with an example. Consider the case of an interpolation scaling function as in section 3.2.2. In that case, a signal $f \in \mathbf{V}_{0}$ is represented by its integer samples $f(n), n \in \mathbb{Z}$, and an element $g \in \mathbf{V}_{1}$ by its even subsamples $g(2 n), n \in \mathbb{Z}$. Following the conditions of theorem 3 , we may take as a product operator over $\mathbf{V}_{j}$ the product of samples at intervals $2^{j}$. The product operator depends on the resolution. Now imagine that we have just computed the product of $N$ signals $x_{1}, \ldots, x_{N}$ in $\mathbf{V}_{1}$, and that we wish to extend this product to the finer resolution $\mathbf{V}_{0}$. This requires using an interpolation formula to compute the values of each $x_{i}$ at the odd positions, and then compute the product of all this values at each of these locations. If the product operator had been causal with respect to the resolutions, all that would have been required would have been an interpolation product on the values of the product in $\mathbf{V}_{1}$ (those at the even positions), which is much simpler.

\section{Making a good resolution}

We study here under which conditions a single resolution $\mathbf{V}_{j}$ can be invariant under a product operator over signals. The product operator may depend here on $j$.

\subsection{The basic shift invariant product is a coor- dinate by coordinate product}

Theorem 2 Assume that $*$ is a non trivially zero associative product operator such that $\left(\mathbf{V}_{j},+, *\right)$ be a commutative ring, that $*$ commutes with the shift of length $2^{j}$, and that the product of two compactly supported functions is zero beyond a certain fixed distance. Then there exist a constant $A \neq 0$ such that, for any $x$ and $y$ in $\mathbf{V}_{j}$, and any $n \in \mathbb{N}$,

$$
<x * y, \phi_{j, n}^{*}>=A<x, \phi_{j, n}^{*}><y, \phi_{j, n}^{*}>\text {. }
$$

Proof: For clarity's sake, we forget the scale index $j$. Let $r$ the maximum integer $n$ for which $\phi_{0} * \phi_{n} \neq 0$; because the product is coomutative, $r \geq 0$. Let $M_{r}=\max \left\{k \in \mathbb{Z} /<\varphi_{0} * \varphi_{r}, \varphi_{k}^{*}>\neq 0\right\}$. Consider now $\left(\phi_{0} * \phi_{r}\right) * \phi_{M_{r}+r}$; there exist a non zero constant $a$ such that it is equal to $a \phi_{M_{r}} * \phi_{M_{r}+r}$, which is itself non zero. On the other hand, it is also equal to $\left(\phi_{0} * \phi_{M_{r}+r}\right) * \phi_{r}$, which is zero if $M_{r}>0$; hence, $M_{r} \leq O$. By a symmetric argument, one verifies that $m_{r}=\min \left\{k \in \mathbb{Z} /<\varphi_{0} * \varphi_{r}, \varphi_{k}^{*}>\neq 0\right\}$ satisfies $m_{r} \geq 0$; hence, $m_{r}=M_{r}=0$. This implies that $\phi_{0} * \bar{\phi}_{r}=A \phi_{0}$. Consider now $\phi_{-r} *\left(\phi_{0} * \phi_{r}\right)=A^{2} \phi_{-r}$; it is also equal to $\left(\phi_{-r} * \phi_{r}\right) * \phi_{0}$, which is non zero if and only if $r=0$.

A consequence of this theorem is that the only resolution spaces that are invariant under the usual function's product are piecewise constant resolutions, which corresponds to the Haar multiresolution. This multiresolution has $p=0$ in theorem 1 , and yields poor approximation orders. The next thing to try is taking the projection of the product instead of the product itself. We shall see in section 3.2.2 that it does not work in the general case and that, in the cases where it works, it not suitable for the definition of multiresolution algebras. This means that we shall have to study alternate product operators that will be approximations of the classical pointwise product (this is relevent, since the objects we multiply are essentially approximations themselves) and, possibly, alternate definitions of a multiresolution (section 4).

\subsection{Approximating product operators}

3.2.1 Caracterization: theorem 1 is generalized to the approximation of a product:

Theorem 3 Assume that $*$ is a product operator such that $\left(\mathbf{V}_{0},+, *\right)$ be a commutative ring, that $*$ commutes with the shift of length $L \in \mathbb{N}$, and that the product of two compactly supported functions is zero beyond a certain fixed distance. We define $*_{j}$ on $\mathbf{V}_{j}$ by rescaling. Let $N \leq p$, with $p$ as in theorem 1. The two following conditions are equivalent:

- For any $[a, b]$ there exist $K$ and $M$ such that, for any functions $x$ and $y$ of class $C^{N+1}$ and any $j \leq 0$,

$$
\begin{gathered}
\sup _{t \in[a, b]}\left|x y-\left(P_{j} x\right) *_{j}\left(P_{j} y\right)\right| \\
\leq K 2^{j(N+1)} \sup _{t \in[a-M, b+M]} \\
\left(\left|x^{(N+1)}(t)\right|+\left|y^{(N+1)}(t)\right|+\left|(x y)^{(N+1)}(t)\right|\right)
\end{gathered}
$$


- the following consistency condition is satified

$$
t^{i} * t^{j}=t^{i+j} \text { if } i+j \leq N
$$

Proof: Necessary condition: define $d=$ $\max \{k \in[0, N] \cap \mathbb{N}$ s.t. $(\forall i \in[0, k] \cap \mathbb{N})(\forall \tau \in[0, L])$ $\left.\left(t^{i} * t^{k-i}\right)(\tau)=\tau^{k}\right\}$. The condition (2) on the order of approximation implies that $d=N$. Sufficient condition: let $x$ and $y$ two smooth, compactly supported functions. Because of theorem 1, we can replace $x$ and $y$ by $P_{j} x$ and $P_{j} y$ in the left handside of (2). Because of the product and projectors are localized, there exists $K$ such that the value $\left(P_{j} x\right) *_{j}\left(P_{j} y\right)(t)$ depends on the value of $x$ and $y$ in $\left(t-K 2^{j}, t+K 2^{j}\right)$. Let $T x$ and $T y$ the Taylor expansions of degree $N$ of $x$ and $y$ at point $t$. We can replace $\left(P_{j} x\right) *_{j}\left(P_{j} y\right)(t)$ by $\left(P_{j} T x\right) *_{j}\left(P_{j} T y\right)(t)$ without degrading the approximation. But it precisely equal to $\left(P_{j} T(x y)\right)(t)$, where $T(x y)$ stands for the Taylor expansion of $x y$ at $t$. The result follows from here.

3.2.2 Lagrange product, interpolating scaling functions and Coiflets: because of theorems 2 and 3, we can see that an approximating product operator commutes with the elementary shift (e.g., $L=1$ ) if and only if

$$
<t^{k}, \phi^{*}>=\left(A<t, \phi^{*}>\right)^{k} \text { for } 1 \leq k \leq \min (p, N) .
$$

This property is not verified by general scaling functions. As a consequence, the projection on a resolution of the classical product, considered as a product operator, is generally not associative (this is the only assumption in theorem 2 which is not verified in that case). Note that Beylkin [1] has introduced a recursive algorithm for computing the wavelet decomposition of the product of two signals. In theory, it requires (in most cases) an infinte number of computations. In practice, it is suggested to truncate the expansion at some resolution $J$. This is equivalent to taking the projection $P_{J}(x y)$ as a product operator. As we have seen, this product is generally not associative; which means that the result depends on the order in which the operations are performed. The product of $n$ signals can have $(n-1)$ ! values, depending on the order. This is not easily manageable.

While (4) is not true in the general case, it is known to be verified by two classes of scaling functions: Coiflets ([3], [2]), which verify $<t^{k}, \phi^{*}>=0$ for $1 \leq k \leq p$, and interpolation scaling functions ([7], [4]), which verify $\phi(0)=0$ and $\phi(n)=0$ for $n \in \mathbb{Z}, n \neq 0$. In that case, we can use (1) as an approximate product operator, which we shall call the Lagrange product.

Theorem 4 Assume that $\left(\mathbf{V}_{0},+, *,.\right)$ be the algebra defined with the Lagrange product (1), and that $p \geq 1$ in theorem 1. Then $\mathbf{V}_{1}$ is not a subalgebra of $\mathbf{V}_{0}$.
Proof: For an arbitrary large integer $N$, we can find an element $u \in \mathbf{V}_{1}$ and an element $m \in \mathbf{V}_{1}$ such that their coordinates in $\mathbf{V}_{0}$ be respectively $u_{n}=1$ and $m_{n}=n$ for $0 \leq n \leq N$. If $\mathbf{V}_{1}$ is invariant by product, then, by using the powers of $m$ and the Lagrange interpolation, we see that we can find an element in $\mathbf{V}_{1}$ with arbitrary coordinates in $\mathbf{V}_{0}$ for indices in $[0, N]$. This contradict the orthogonality of $\mathbf{V}_{1}$ and $\mathbf{W}_{0}$, which is expressed by a zero output through a given finite impulse response filter.

Anticipating the results of section 4, we see that the Lagrange product is unable to provide a product which is causal with respect to the multiresolution.

3.2.3 Construction of approximating products for general scaling functions: In theorem 3, we have caracterized approximate product operators over $\mathbf{V}_{0}$. There remains to build some of these, beside the Lagrange product that theorem 4 has shown to be improper for multiresolution algebras. This is done by identifying local polynomials in the coordinate space and then using theorem 3 through condition (3).

Theorem 5 Let $\left(x_{0}, \ldots, x_{q}\right)$ a sequence of $q+1$ real numbers. Then, for any $j \in \mathbb{N}$, there exists a unique polynomial $Q$ of degree $\leq q$ such that

$$
x_{k}=<Q, \phi_{j, k}^{*}>\text { for all } 0 \leq k \leq q
$$

Proof: Define the matrix $A=\left(a_{i, j}\right)$ with $a_{k, i}=<$ $t^{i}, \varphi_{k}^{*}>=<(t+k)^{i}, \varphi_{0}^{*}>$ and $B=\left(b_{i, j}\right)$ with $b_{i, j}=$ $\left(\begin{array}{c}j-1 \\ i-1\end{array}\right)$ if $i \leq j$ and $b_{i, j}=0$ if $i>j . B$ is invertible, and the matrix that changes the basis $(1,(t+k), \ldots(t+$ $\left.k)^{q}\right), k \in \mathbb{Z}$, into $\left(1, t, \ldots t^{q}\right)$ is equal to $B^{k}$. If we denote by $M$ the vector matrix of the $q+1$ first moments of $\varphi^{*}$, then we have:

$$
A=\left[\begin{array}{cccc}
M^{T} & 0 & \cdots & 0 \\
0 & \ddots & \ddots & \vdots \\
\vdots & \ddots & \ddots & 0 \\
0 & \cdots & 0 & M^{T}
\end{array}\right]\left[\begin{array}{c}
\mathrm{Id} \\
B \\
\vdots \\
B^{q}
\end{array}\right]
$$

Let us now denote by $\mathcal{B}$ a matrix that is obtained from $B$ by substituting each of its element by the outer product of it with the identity matrix that is the size of $B$. Then we have

$$
\left[\begin{array}{c}
\mathrm{Id} \\
B \\
\vdots \\
B^{q}
\end{array}\right]=\mathcal{B}^{T}\left[\begin{array}{c}
\mathrm{Id} \\
B-\mathrm{Id} \\
\vdots \\
(B-\mathrm{Id})^{q}
\end{array}\right]
$$

Observe that $(B-\mathrm{Id})^{k}$ has its first $k$ columns equal to zero (as well as its last $k$ rows). On the other hand, 
we can see that the product of the large $M^{T}$-diagonal matrix by $\mathcal{B}^{T}$ is in fact equal to $B^{T} M^{T}$, so that we have, in the end:

$$
A=B^{T} M^{T}\left[\begin{array}{c}
\mathrm{Id} \\
B-\mathrm{Id} \\
\vdots \\
(B-\mathrm{Id})^{q}
\end{array}\right] \stackrel{\text { def }}{=} B^{T} M^{T} B_{-}
$$

Observe that the product of $M^{T}$ with the large matrix $B_{-}$on the right is upper triangular, with the first (and non zero) moment of $\phi^{*}$ on the diagonal. Since $B$ is regular, $A$ has full rank.

Let $\mathbb{R}_{q}[t]$ the space of real polynomials with degree $\leq q$. Thanks to the previous mapping, we can identify any element of $\mathbf{V}_{j}$ to a sequence of elements of $\mathbb{R}_{q}[t]$. We shall denote by $\Pi$ this mapping from $\mathbf{V}_{j}$ into $\left(\mathbb{R}_{q}[t]\right)^{\mathbb{Z}}$. Observe that, if $t^{k} \in \mathbf{V}_{j}$ and $k \leq q$, $\Pi t^{k}$ is the sequence $p_{n}(t)=\left(n 2^{j}+t\right)^{k}$; we shall use this in section 4 .

To construct a product operator on $\mathbf{V}_{j}$ that satisfies the conditions of theorem 3 , it is sufficient to contruct a product on $\mathbb{R}_{q}[t]$ which verifies (3), extend it to $\left(\mathbb{R}_{q}[t]\right)^{\mathbb{Z}}$, and map it back to $\mathbf{V}_{j}$. The following theorem describes product operators on $\mathbb{R}_{q}[t]$ which satisfy (3).

Theorem 6 Let $*$ an associative, commutative product over $\mathbb{R}_{q}[t]$ which satifies (3), and define $Q \in \mathbb{R}_{q}[t]$ by $Q=t^{q} * t$. Then, for any $x$ and $y$ in $\mathbb{R}_{q}[t], x * y$ is the Hermite interpolation of $x y$ at the (possibly multiple) zeros of $t^{q+1}-Q(t)$ in $\mathbb{C}$. Conversely, any such product is associative, commutative, and satisfies (3).

Proof: one can verify recursively that the interpolation property is satisfied by $T_{n+1} \stackrel{\text { def }}{=} T_{n} * T$, with $T_{0}=1$.

The Lagrange product corresponds to the case where all the zeros are distinct. In that case, the polynomial identification is not necessary to compute the product, because it does not involve any derivative. In the other cases, the knowledge of the underlying polynomial is required.

By extending an Hermite interpolating product $*$ to $\left(\mathbb{R}_{q}[t]\right)^{\mathbb{Z}}$, and using $\Pi^{-1}$ to map it back to $\mathbf{V}_{j}$, an approximate product on $\mathbf{V}_{j}$ is constructed, whatever the scaling functions, and for any $j$. Indeed, this product satisfies the assumptions of theorem 3 with $L=q+1$ and $N=\min (p, q)$. Observe that the product operator depends on $j$. In the next section we study how one can build a multiresolution algebra atop a single resolution $\mathbf{V}_{J}$ endowed with such an Hermite approximating product.

\section{Climbing up the scales}

As we have seen, the Lagrange product does not allow causal multiresolution algebras. But we can study multiresolutions under the polynomial representation instead of the functional representation $\mathbf{V}_{J}$, or of the scaling coefficient representation $\left\langle x, \phi_{J, n}^{*}\right\rangle, n \in \mathbb{Z}$. This allows the use of the general Hermite interpolating products of theorem 6 . To study what can be a piecewise polynomial multiresolution algebra, we start by studying the subalgebras of $\left(\mathbb{R}_{q}[t]\right)^{I}$ with $I \subset \mathbb{Z}$ and $I$ finite.

\subsection{Subalgebras of $\left(\mathbb{R}_{q}[t]\right)^{I}$}

We relate the dimension of subalgebras to the overlapping interpolation points, and build a subalgebra of minimum size from these. To that end, we denote by $\mathcal{Z}$ the set of interpolating points $z$ as in theorem 6 , without their order of multiplicity $o(z)$.

Lemma 1 Define $S_{1}$ as the subspace of sequences $\left(p_{k}\right)$ in $S$ such that, $p_{k}^{(j)}\left(z+2^{J} k\right)=p_{\tilde{k}}^{(j)}\left(\tilde{z}+2^{J} \tilde{k}\right)$ if $z+$ $2^{J} k=\tilde{z}+2^{J} \tilde{k}$ and $j<\min (o(z), o(\tilde{z}))$. Then $S_{1}$ is a subalgebra of $S$.

Proof: The nature of the product operator garantees that the constraints that define $S_{1}$ are preserved by product.

$S_{1}$ is defined for any $I \subset \mathbb{Z}$. For $I=\mathbb{Z}, S_{1}$ includes the images $\Pi t^{k}$ of the polynomials such that $k \leq \min (p, q)$. This is actually the reason why we take a shift of $2^{J}$ in the definition of $S_{1}$.

For $i \in I$, we define $\mathcal{Z}_{i}=\mathcal{Z}+i 2^{J}, \mathcal{Z}_{I}=\cup_{i \in I} \mathcal{Z}_{i} . z_{i} \in \mathcal{Z}_{i}$ and $z_{j} \in \mathcal{Z}_{j}$ overlap if they have the same value. For an element $z \in \mathcal{Z}_{I}, I(z)$ is the set of integer $i \in I$ such that a (necessarily unique) element $z_{i}(z)$ of $\mathcal{Z}_{i}$ is equal to $z$. For $z \in \mathcal{Z}_{I}$ we define $\omega(z)=\max _{i \in I(z)}\left\{o\left(z_{i}(z)\right)\right\}$, and $\Omega=\sum_{z \in \mathcal{Z}_{I}} o(z)$. The following lemma gives an interpretation of $S_{1}$ by relating it to an algebra of "global" polynomials.

Lemma 2 Consider $\mathbb{R}_{\Omega}[t]$ with the the product defined by Hermite interpolation at the points $z \in \mathcal{Z}_{I}$ at order $\omega(z)$. For any $p \in \mathbb{R}_{\Omega}[t]$, define $H p$ as the sequence $H p=\left(p_{k}\right)_{k \in I}$ with $p_{k}^{(j)}(z)=p^{(j)}\left(z-k 2^{J}\right)$ for any $z \in \mathcal{Z}$ and any $0 \leq j \leq o(z)$. Then $H$ is an algebraic isomorphism between $\mathbb{R}_{\Omega}[t]$ and $S_{1}$.

Proof: It can be verified that $H p$ does belong to $S_{1}$. From the definition of $S_{1}$, we see that $H$ is indeed one to one.

This lemma shows that the elements of $S_{1}$ can be represented as one polynomial of degree $<\Omega$. Observe 
that, for $I=\mathbb{Z}$ and $k \leq q$, the image $\Pi H t^{k}$ of $t^{k} \in \mathbf{V}_{J}$ is precisely $t^{k} \in \mathbb{R}_{\Omega}[t]$.

Theorem 7 Let $S$ a subalgebra of $\left(\mathbb{R}_{q}[t]\right)^{I}$, and assume that $S$ contains the constant sequence, $T_{0}=$ $(1)_{i \in I}$, and the linear sequence, $T_{1}=\left(i 2^{J}+t\right)_{i \in I}$. Then $S_{1} \subset S$.

Proof: $\quad T_{0} \in S_{1} \cap S$ and $T_{1} \in S_{1} \cap S$; hence the powers $T_{n} \stackrel{\text { def }}{=} T_{n-1} * T_{1}$ belong to $S_{1} \cap S$. On the other hand, $H^{-1} T_{n}=t^{n}$ if $n<\Omega$. This proves that $\left(T_{0}, T_{1}, \ldots, T_{\Omega-1}\right)$ is a basis of $S_{1}$, and that $S_{1} \subset S$.

As a consequence, $S_{1}$ is a minimal subalgebra for all algebras that include the constant and linear sequences. Its dimension is $\Omega$. If the number of elements of $I$ is an even number $2 n$, then $\left(\mathbb{R}_{q}[t]\right)^{I}$ is dimension $2 n(q+1)$, and one can verify that $\Omega>n(q+1)$ by studying the set of possible overlapping interpolation points. This implies that a subalgebra of $\left(\mathbb{R}_{q}[t]\right)^{[0,2 n-1]}$ cannot be obtained by a rescaling method similar to the method used to define the resolutions in definition 1 , even in the polynomial representation, because it produces a subspace of dimension $n(q+1)<\Omega$.

$\Omega$ is minimized by maximizing the number of overlapping zeroes. Assuming that the distance between the elements of $\mathcal{Z}$ does not exceed the shift $2^{J}$, this maximisation is achieved by taking $q$ odd and taking two interpolation points at a distance $2^{J}$ and a multiplicity of $(q+1) / 2$. For $I=\mathbb{Z}$, and this interpolation choice, $S_{1}$ is called a Hermite function space. It is the space of piecewise polynomial functions with degree $\leq q$ and which are of class $C^{(q-1) / 2}$ at the nodes $n 2^{J}, n \in \mathbb{Z}$. Hermite function spaces are known in numerical analysis for their good interpolation properties and for giving simple composite integration rules [8].

\subsection{Algebraic multiresolution approximations}

Now that the subalgebras of $\left(\mathbb{R}_{q}[t]\right)^{I}$ have been studied, one may wonder if they have some approximation properties.

Theorem 8 Assume that $S$ is an subalgebra of $\left(\mathbb{R}_{q}[t]\right)^{\mathbb{Z}}$ which include constant and linear sequences as in theorem 7 with $I=\mathbb{Z}$, and that $q \geq p$. Then $\Pi^{-1} S$ is a subalgebra of $\left(\mathbf{V}_{J},+, *,.\right)$ which generates polynomials of degree smaller or equal to $p$.

Proof: We have seen that $S_{1}$ includes $\Pi t^{k}$ for $0 \leq$ $k \leq \min (p, q)$.

Assuming that a projection from $\left(\mathbb{R}_{q}[t]\right)^{\mathbb{Z}}$ into $S$ has been defined, and that the projection and $S$ commute with some shift of length $M$, it corresponds to a projection from $\mathbf{V}_{J}$ into $\Pi^{-1} S$ which commutes with shifts of length $(q+1) M 2^{J}$. Thanks to the general version of theorem 1 [9], this implies that it approximates smooth compacty supported functions with an error of order $\left((q+1) M 2^{J}\right)^{p+1}$. The product is approximated with a similar error.

Example of an algebraic multiresolution approximation $q$ is taken odd with $q=p$, the interpolation points are 0 and $2^{J}$ with both orders $(q+1) / 2 . S_{1}$ is then a Hermite function space. The multiresolution $\left(\mathbf{H}_{j}\right)_{j \geq 0}$ is defined with $\mathbf{H}_{0}=\mathbf{V}_{J}$, and $\mathbf{H}_{j}$ is the set of $x \in \mathbf{H}_{0}$ such that, when $n \in \mathbb{Z}$ is not a multiple of $2^{j}$, the identity

$$
(\Pi x)_{n-1}^{(k)}\left(2^{J}\right)=(\Pi x)_{n}^{(k)}(0) \text { for } 0 \leq k \leq \frac{q-1}{2}
$$

is satisfied. A projection from $\Pi \mathbf{H}_{0}$ on $\Pi \mathbf{H}_{j}$ can be defined by taking the average of $(\Pi x)_{n-1}^{(k)}\left(2^{J}\right)$ and of $(\Pi x)_{n}^{(k)}(0)$ at the points $n \neq k 2^{j}, \forall k \in \mathbb{Z}$. Going from $\Pi \mathbf{H}_{j}$ into $\Pi \mathbf{H}_{j+1}$ amounts to smoothing one out of every two remaining singularity nodes. Such a projection garantees a linear approximation of order $\left((q+1) 2^{J+j}\right)^{p+1}$, and a similar product approximation. This proves that this multiresoltion is indeed an sequence of approximation algebras.

Where are the wavelets? Since classical rescaling is not used anymore to define the resolutions, this implies that wavelets, strictly speaking, have no use anymore. However, since multiresolutions can be defined, this implies the existence of detailed spaces. As in the classical theory of wavelets, the choice of detail spaces is not unique and depends on the practical use of the decomposition.

For instance, it is possible, using cubic Hermite function spaces, to have detail spaces such that the product of a detail signal with a smoother resolution signal is a detail signal with a similar singularity distribution. This is an extension of what happens in the case of the Haar basis. To that end, the basis of the detail space is defined by two pairs of polynomials. Both pairs have a zero value at the leftmost and rightmost boundaries. The first pair has two zero derivatives and opposite, non zero values at the middle interpolation point. The other pair has zero values at the midpoint and opposite derivatives. This solution corresponds to the previous projection, which performs an average of the values of the signal and of its derivative. There is also the possibility, in the same case, to define an $\mathrm{E}^{2}$ theory with an ad hoc approximation of the integration operator and of the nonnegativity of functions. The first solution seems more adapted to the study of static nonlinearities, while the second one seems to suit the dynamical case better; it also restores the property of the vanishing moments. Observe that, nonetheless, the nullity of the moments is not anymore an necessity for studying the smoothness of signals, since this directly handled 
by the structure of the multiresolution.

\section{References}

[1] Beylkin G., On the fast algorithm for multiplication functions in the wavelet bases, Proceedings of the International Conference "Wavelets and Applications", Toulouse, 1992, Y. Meyer and S. Roques, edt., Éditions Fontières, 1993, available at

ftp://amath.colorado.edu

/pub/wavelets/papers/malgToulouse.ps.Z.

[2] Daubechies I., Ten Lectures on Wavelets, CBMSNSF Regional Conference Series in Applied Mathematics, Society for Industrial and Applied Mathematics, 1992.

[3] Daubechies I., Orthonormal bases of compactly supported wavelets, Commun. on Pure and Appl. Math., 41:909-996, November 1988.

[4] Mallat S., A Wavelet Tour of Signal Processing, Academic Press, 1998.

[5] Mallat S., Multiresolution approximations and wavelet orthonormal bases of $\mathbf{L}^{\mathbf{2}}(\mathbb{R})$, Trans. Amer. Math. Soc., 315:69-87, September 1989.

[6] Meyer Y., Wavelets and Operators, Advanced Mathematics, Cambridge University Press, 1992.

[7] Saito N. and Beylkin G., Multiresolution representation using the auto-correlation functions of compactly supported wavelets, IEEE Trans. on Signal Proc., 41(12):3584-3590, December 1993.

[8] Stoer J. and Burlisch R., Introduction to Numerical Analysis, second edition, pp. 52-57 and 126-131, Springer-Verlag, 1993.

[9] Strang G. and Fix G., A Fourier analysis of the finite element variational method, Construct. Aspects of Funct. Anal., pp. 796-830, 1971. 